\title{
Field-Theoretic Simulation of Block Copolymers at Experimentally Relevant Molecular Weights
}

\author{
Bart Vorselaars, ${ }^{* \dagger}$ Pawel Stasiak, ${ }^{\dagger}$ and Mark W. Matsen ${ }^{*} \dagger$ \\ ${ }^{\dagger}$ Department of Chemical Engineering, Department of Physics \& Astronomy, and the Waterloo Institute for Nanotechnology, \\ University of Waterloo, Waterloo, Ontario N2L 3G1, Canada \\ ${ }^{\ddagger}$ School of Mathematical and Physical Sciences, University of Reading, Whiteknights, Reading RG6 6AX, U.K.
}

ABSTRACT: Field-theoretic simulation (FTS) offers an efficient means of predicting the equilibrium behavior of high-molecular-weight structured polymers, provided one is able to deal with the strong ultraviolet (UV) divergence that occurs at realistic molecular weights. Here melts of lamellar-forming diblock copolymer are studied using a Monte Carlo version (MC-FTS), where the composition field fluctuates while the pressure field follows the mean-field approximation. We are able to control the UV divergence by introducing a new effective FloryHuggins interaction parameter, $\chi_{\mathrm{e}}$, thereby permitting MC-FTS for molecular weights extending down to values characteristic of experiment. Results for the disordered-state structure function, the layer spacing and compressibility of the ordered lamellar phase, and the position of the order-disorder transition (ODT) show excellent agreement with recent particle-based simulation. Given the immense versatility of FTS, this opens up the opportunity for quantitative studies on a wide range of more complicated block copolymer systems.

\section{INTRODUCTION}

Block copolymers, formed by joining together at least two chemical distinct subchains (generally labeled A and B), are renowned for their ability to self-assemble into a myriad of ordered morphologies. ${ }^{1}$ Interest in block copolymers has exploded in recent years with the development of cost-effective methods of synthesizing these molecules combined with an ever-growing list of applications, such as thermoplastic elastomers, compatibilizers, adhesives, lithography, porous materials, and photonic crystals. ${ }^{2}$ Progress has been greatly aided by a thorough understanding of their equilibrium behavior due in large part to self-consistent-field theory (SCFT).$^{3-5}$ The theory is based upon a standard Gaussianchain model, ${ }^{6}$ where the polymer chains are treated as thin elastic threads interacting by a simple contact force, the strength of which is controlled by a Flory-Huggins interaction parameter, $\chi$. The model is typically solved in the incompressible limit, where the total concentration of the A and $\mathrm{B}$ components is uniform.

To perform the statistical mechanics of this particle-based model, one introduces mathematical identities involving integrals over auxiliary fields. This replaces the particle-particle interactions with particle-field interactions, which in turn allows one to integrate (or sum) over all the polymer coordinates. The result is an effective Hamiltonian corresponding to a field-theoretic model involving just the auxiliary fields. In SCFT, the sum over all possible field configurations is performed by a saddle-point approximation, which equates to mean-field theory. This works well for ordered morphologies

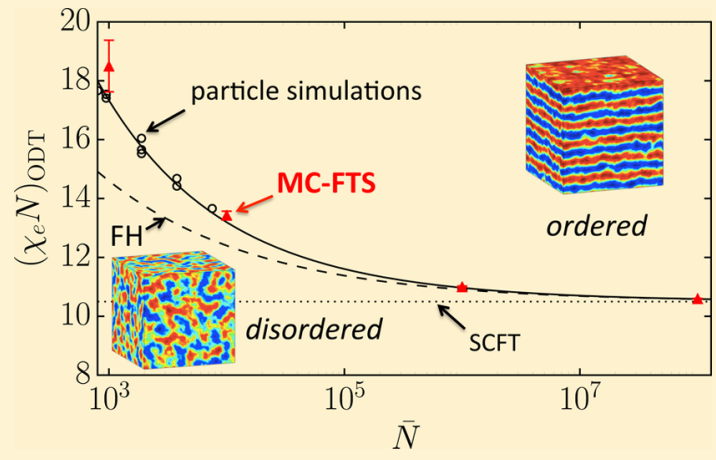

but fails for disordered phases. In particular, SCFT treats the disordered phase as a perfectly homogeneous mixture of the unlike components, when in fact the composition (the difference in the A and B concentrations) generally involves significant fluctuations.

The standard testing ground for fluctuation effects is the symmetric diblock copolymer, where half its $N$ segments form the A block and the remaining ones form the B block. According to mean-field theory, ${ }^{3}$ a neat melt of these molecules undergoes a continuous order-disorder transition (ODT) to a lamellar phase when the product $\chi N$ exceeds 10.495. In 1987, Fredrickson and Helfand derived a theory to account for the fluctuations, ${ }^{8}$ predicting a discontinuous ODT at

$$
(\chi N)_{\mathrm{ODT}}=10.495+41.0 \bar{N}^{-1 / 3}
$$

A more refined calculation by Mayes and Olvera de la Cruz ${ }^{9}$ gives a coefficient of 39.0 instead of 41.0. In any case, the strength of fluctuation effects depends on the invariant polymerization index, $\bar{N}=a^{6} \rho_{0}{ }^{2} N$, where $a$ is the statistical segment length and $\rho_{0}$ is the bulk segment density. SCFT is exact for infinitely long polymers, but fluctuation effects become increasingly important as $\bar{N}$ decreases. For realistic experiments, ${ }^{10} \bar{N} \approx 10^{2}-10^{4}$, but the approximations of the FH theory are only strictly valid for $\bar{N} \gtrsim 10^{10}$. Nevertheless, it has remained the dominant fluctuation theory for nearly 30 years

Received: October 17, 2015

Revised: December 2, 2015

Published: December 11, 2015 
because its predictions agree qualitatively with experiment and there has been no viable alternative. Morse and co-workers ${ }^{11-13}$ have developed a more sophisticated treatment, renormalized one-loop (ROL) calculations, ${ }^{14}$ for examining fluctuations in the disordered state, but predictions for the ODT have not been possible because of the difficulty in dealing with ordered phases.

In lieu of a more accurate and tractable theory, simulation $^{15,16}$ offers a useful way forward. However, one of the challenges of conventional particle-based simulations is mapping the model parameters onto the standard block copolymer model involving $\chi$. This difficulty was recently resolved by Glaser et al. ${ }^{17}$ by using the disordered-state structure function predicted by ROL, $S_{\mathrm{ROL}}(k)$, as a means of defining an effective $\chi$ parameter. In doing so, they were able to collapse the ODTs of five distinct models onto a common empirical curve ${ }^{18}$

$$
(\chi N)_{\mathrm{ODT}}=10.495+41.0 \bar{N}^{-1 / 3}+123.0 \bar{N}^{-0.56}
$$

which assumes the $\mathrm{FH}$ prediction (eq 1) in the large- $\bar{N}$ limit. Because of the computational cost of simulating highmolecular-weight polymers, it remains difficult to access the ODT at large values of $\bar{N}$.

A promising strategy for handling larger molecules is fieldtheoretic simulation (FTS), whereby the statistical mechanics of the field-theoretic model from SCFT are simulated rather than evaluated with the saddle-point approximation; ref 19 provides a nice review of FTS. For the simple diblock, the effective Hamiltonian involves a composition field, $W_{-}(\mathbf{r})$, that couples to the difference in the A and B concentrations and a pressure field, $W_{+}(\mathbf{r})$, that enforces incompressibility. The difficulty is that in the mathematical transformation to fields $W_{+}(\mathbf{r})$ takes on imaginary values resulting in a complex-valued Hamiltonian. Fredrickson et al. ${ }^{19}$ have dealt with this by employing complex-Langevin ${ }^{20}$ simulations (CL-FTS). FTS is effective at large $\bar{N}$, but it struggles to cope with $\bar{N}$ values characteristic of real experiments. So far, applications of CLFTS to the standard Gaussian-chain model for diblock copolymer melts in three dimensions (3D) have been limited to $\bar{N}=5.4 \times 10^{5},{ }^{21,22}$ which is well above the experimental range. Schmid and co-workers ${ }^{21,23}$ tackled the problem by introducing an approximation to FTS, where $W_{-}(\mathbf{r})$ is allowed to fluctuate but $W_{+}(\mathbf{r})$ follows the saddle-point, $w_{+}(\mathbf{r})$, corresponding to a mean-field treatment of the incompressibility condition. The main advantage is that $w_{+}(\mathbf{r})$ is real valued, which allows for conventional Monte Carlo simulations (MCFTS). It is reasonable to expect the saddle-point approximation for $W_{+}(\mathbf{r})$ to be accurate, and indeed there are a couple studies indicating this to be true. ${ }^{21,24}$

Stasiak and Matsen ${ }^{25}$ have managed to perform 3D MC-FTS down to $\bar{N}=10^{4}$, which is tantalizingly close to the experimental regime. In doing so, however, they encountered an ultraviolet (UV) divergence, where increasing the mesh resolution (thereby allowing shorter wavelength fluctuations) tends to disorder the melt. The presence of this divergence was previously anticipated by Olvera de la Cruz et al. ${ }^{26}$ back in 1988. They showed that it could be compensated for by increasing the bare interaction parameter, $\chi_{\mathrm{b}}$, or equivalently by defining an effective interaction parameter, $\chi_{\mathrm{e} 1}$. Having controlled the UV divergence, Stasiak and Matsen found reasonable agreement with the FH prediction (eq 1) for $\bar{N}=$ $10^{4}-10^{8}$. This initial study, however, was limited to relatively small simulation boxes with fixed dimensions, and so results may have been somewhat tainted by finite-size effects.

Here we take on the challenge of performing accurate FTS at experimentally relevant molecular weights (e.g., $\bar{N}=10^{3}$ ). In our attempts to do so, we find that the $\chi_{\mathrm{el}}$ derived by Olvera de la Cruz et al. fails to control the UV divergence at small $\bar{N}$, but fortunately we are able to circumvent the problem by deriving an alternative interaction parameter, $\chi_{\mathrm{e} 2}$. We also reduce finitesize effects by using GPUs to simulate larger systems and by including a Monte Carlo move that allows the lamellar period to equilibrate. With these advances, we obtain a wealth of new results that match up nicely with those of recent particle-based simulations. ${ }^{18,27}$

\section{THEORY}

Model and Simulation Method. Our study examines the standard Gaussian-chain model ${ }^{4}$ for an incompressible melt of $n$ $\mathrm{AB}$ diblock copolymers in a fixed volume, $V=n N / \rho_{0}=n / \rho_{c}$, where $\rho_{\mathrm{c}}$ is the chain density. The partition function for this model is

$$
Z \sim \int \exp \left(-\frac{H_{\mathrm{p}}\left[\left\{\mathbf{r}_{\alpha}\right\}\right]}{k_{\mathrm{B}} T}\right) \delta\left[\hat{\phi}_{\mathrm{A}}+\hat{\phi}_{\mathrm{B}}-1\right] \prod_{\alpha=1}^{n} \mathcal{D} \mathbf{r}_{\alpha}
$$

where the integration runs over the conformations, $\mathbf{r}_{\alpha}(s)$, of each polymer chain $\alpha$ with its contour parametrized by $s$. The delta function ensures the incompressibility of the combined Asegment concentration

$$
\hat{\phi}_{\mathrm{A}}(\mathbf{r})=\frac{1}{\rho_{\mathrm{c}}} \sum_{\alpha=1}^{n} \int_{0}^{f} \delta\left(\mathbf{r}-\mathbf{r}_{\alpha}\right) \mathrm{d} s
$$

and B-segment concentration, $\hat{\phi}_{\mathrm{B}}(\mathbf{r})$, given by an analogous expression with $s$ integrated from $f$ to 1 . The particle-based (i.e., segment-based) Hamiltonian is given by

$$
\frac{H_{\mathrm{p}}\left[\left\{\mathbf{r}_{\alpha}\right\}\right]}{k_{\mathrm{B}} T}=\chi_{\mathrm{b}} N \rho_{\mathrm{c}} \int \hat{\phi}_{\mathrm{A}} \hat{\phi}_{\mathrm{B}} \mathrm{d} \mathbf{r}+\frac{3}{2 R_{0}^{2}} \sum_{\alpha=1}^{n} \int_{0}^{1}\left|\mathbf{r}_{\alpha}^{\prime}\right|^{2} \mathrm{~d} s
$$

where the first term accounts for the interactions between A and $\mathrm{B}$ segments controlled by the bare Flory-Huggins parameter, $\chi_{\mathrm{b}}$, and the second term accounts for chain stretching controlled by the end-to-end length of an unperturbed polymer, $R_{0}=a N^{1 / 2}$.

In SCFT and FTS, the segment-segment interactions of $H_{\mathrm{p}}\left[\left\{\mathbf{r}_{\alpha}\right\}\right]$ are decoupled via the introduction of fields, ${ }^{5,21,28}$ which allows one to integrate over the particle coordinates, $\left\{\mathbf{r}_{\alpha}\right\}$. The result is a mathematically equivalent field-based Hamiltonian

$$
\frac{H_{\mathrm{f}}\left[W_{-}, W_{+}\right]}{k_{\mathrm{B}} T}=-n \ln Q+\rho_{\mathrm{c}} \int\left(\frac{W_{-}^{2}}{\chi_{\mathrm{b}} N}-W_{+}\right) \mathrm{d} \mathbf{r}
$$

where $Q\left[W_{-}, W_{+}\right]$is the partition function for a single chain under the influence of the fields, $W_{-}(\mathbf{r})$ and $W_{+}(\mathbf{r})$. Rather than integrating over fluctuations in the pressure field, MC-FTS applies the saddle-point approximation (i.e., $W_{+} \rightarrow w_{+}$), ${ }^{21,25}$ whereby the partition function

$$
Z \sim \int \exp \left(-\frac{H_{\mathrm{f}}\left[W_{-}, w_{+}\right]}{k_{\mathrm{B}} T}\right) \mathcal{D} W_{-}
$$


involves a single integration over $W_{-}(\mathbf{r})$, which is performed using the standard Metropolis MC algorithm.

Details of the numerical techniques are given in ref 25 . In short, there are two computational challenges to deal with. The first is the evaluation of $Q$ which requires solving a modified diffusion equation in an orthorhombic box of lengths $L_{\nu}(\nu \in$ $\{x, y, z\})$ with periodic boundary conditions. This is done using a pseudospectral algorithm, which involves transforming back and forth between a real-space grid with $m_{\nu}=L_{\nu} / \Delta_{\nu}$ points spaced by $\Delta_{\nu}$ along each Cartesian axis, $\nu$, and a reciprocalspace lattice with wavevectors $\mathbf{k}=\left(k_{x}, k_{y}, k_{z}\right)$ given by $k_{\nu}=2 \pi j_{\nu} /$ $L_{\nu}$ with $j_{\nu} \in\left\{-m_{\nu} / 2, \ldots,\left(m_{\nu}-1\right) / 2\right\}$. (Note that we omit the subscript $\nu$ when quantities are the same for all three axes.) The fast Fourier transforms for our smaller system sizes (e.g., $m \lesssim$ 24) are calculated on central-processing units (CPUs), while for larger systems we use graphics processor units (GPUs). ${ }^{29}$ The other challenge is locating the saddle point, $w_{+}(\mathbf{r})$, for which we employ the Anderson mixing scheme in ref 25. In the current study, however, a more stringent error tolerance of $\epsilon=$ $10^{-4}$ is imposed.

Effective Interaction Parameter. Ideally, FTS results would become independent of the spatial grid at a sufficiently fine resolution, but this is prevented by the UV divergence mentioned above. In ref 25 , the divergence was compensated for by expressing results in terms of an effective interaction parameter originally derived by Olvera de la Cruz et al. ${ }^{26}$

$$
\chi_{\mathrm{e} 1}=\left(1-\frac{6 \alpha}{\pi^{2}} l \Lambda\right) \chi_{\mathrm{b}}
$$

where $l=R_{0} / \sqrt{ } \bar{N}=1 /\left(\rho_{0} a^{2}\right)$ is the invariant segment length (or packing length) and $\Lambda$ specifies the cutoff of large wavevectors. For the spherical cutoff (i.e., $|\mathbf{k}| \leq \Lambda$ ) used in the original derivation, $\alpha=1$, while for the cubic cutoff (i.e., $k_{\nu}$ $\leq \Lambda=\pi / \Delta)$ used in the MC-FTS of Stasiak and Matsen, $\alpha=$ $1.221 .^{25}$ Here we find that this renormalization of $\chi$ does not suffice for the small $\bar{N}$ this study is aiming to simulate, which leads us to derive an alternative expression in the spirit of the $\chi_{\mathrm{e}}$ defined by Müller and Binder $^{30}$ for a lattice model of a homopolymer blend.

Our starting point is as in ref 26, the free energy, F. Its dependence on a parameter controlling the energy of mixing ${ }^{31}$ (e.g., $\chi$ ) can be determined by thermodynamic integration ${ }^{16}$

$$
F(\chi)-F(0)=\int_{0}^{\chi} \frac{\partial F}{\partial \chi} \mathrm{d} \chi=\int_{0}^{\chi}\left\langle\frac{\partial H}{\partial \chi_{\mathrm{b}}}\right\rangle\left(\frac{\partial \chi}{\partial \chi_{\mathrm{b}}}\right)^{-1} \mathrm{~d} \chi
$$

Referring to eq 5, the thermodynamic average

$$
\left\langle\frac{\partial H}{\partial \chi_{\mathrm{b}}}\right\rangle=k_{\mathrm{B}} T \rho_{0} \int\left\langle\hat{\phi}_{\mathrm{A}} \hat{\phi}_{\mathrm{B}}\right\rangle \mathrm{d} \mathbf{r}
$$

is proportional to the total number of $\mathrm{A}-\mathrm{B}$ contacts. In the absence of composition fluctuations, $\left\langle\hat{\phi}_{\mathrm{A}}(\mathbf{r}) \hat{\phi}_{\mathrm{B}}(\mathbf{r})\right\rangle=$ $\left\langle\hat{\phi}_{\mathrm{A}}(\mathbf{r})\right\rangle\left\langle\hat{\phi}_{\mathrm{B}}(\mathbf{r})\right\rangle=f(1-f)$ for the disordered phase, and thus its free energy reduces to the Flory-Huggins expression ${ }^{26,32}$

$$
F(\chi)-F(0)=k_{\mathrm{B}} T \rho_{0} V f(1-f) \chi
$$

corresponding to mean-field theory (i.e., SCFT). However, if fluctuations are present, then

$$
\begin{aligned}
\int\left\langle\hat{\phi}_{\mathrm{A}} \hat{\phi}_{\mathrm{B}}\right\rangle \mathrm{d} \mathbf{r} & =V f(1-f)-\int\left\langle\delta \hat{\phi}_{\mathrm{A}}(\mathbf{r}) \delta \hat{\phi}_{\mathrm{A}}(\mathbf{r})\right\rangle \mathrm{d} \mathbf{r} \\
& =V f(1-f)-\frac{1}{(2 \pi)^{3}} \int\left\langle\delta \hat{\phi}_{\mathrm{A}}(\mathbf{k}) \delta \hat{\phi}_{\mathrm{A}}(-\mathbf{k})\right\rangle \mathrm{d} \mathbf{k} \\
& =V f(1-f)\left(1-\frac{\int S(\mathbf{k}) \mathrm{d} \mathbf{k}}{(2 \pi)^{3} \rho_{\mathrm{c}} \rho_{0} N f(1-f)}\right)
\end{aligned}
$$

where the second and third expressions are obtained using the Fourier representation of the composition profile and the definition of the structure function, $S(\mathbf{k})$, respectively. As proposed by Müller and Binder, we require that eq 9 reduces to eq 11 in the athermal limit (i.e., $\chi \rightarrow 0$ ), which is achieved by equating $\chi$ to

$$
\chi_{\mathrm{e} 2}=\left(1-l \frac{R_{0}^{2} \int S_{\mathrm{RPA}, 0}(k) \mathrm{d} \mathbf{k}}{(2 \pi)^{3} \rho_{0} N f(1-f)}\right) \chi_{\mathrm{b}}
$$

This expression makes use of the fact that the structure function in MC-FTS reduces to that of the random-phase approximation $(\mathrm{RPA})^{3}$ in the $\chi \rightarrow 0$ limit. For the regular grid used in this study, the integration is constrained to an orthorhombic box, $k_{\nu} \leq \pi / \Delta_{\nu}$ for $\nu \in\{x, y, z\}$. In practice, however, we approximate the integral, $\int S_{\mathrm{RPA}, 0}(k) \mathrm{dk}$, by the sum $(2 \pi)^{3} V^{-1} \sum_{\mathbf{k}} S_{\mathrm{RPA}, 0}(k)$. Note that eq 8 is recovered by simply substituting the large- $k$ approximation of the structure function

$$
\frac{S_{\mathrm{RPA}, 0}(k)}{\rho_{0} N} \rightarrow \frac{12 f(1-f)}{k^{2} R_{0}^{2}} \text { as } k \rightarrow \infty
$$

into eq 13 , which ensures that the new expression agrees with the previous one for large $\bar{N}$.

Box-Altering MC Move. To treat the ordered lamellar phase accurately, it is necessary for the simulation box to be commensurate with the equilibrium lamellar period. To achieve this, we introduce a Monte Carlo move employed by Zong and $\mathrm{Wang}^{33}$ for particle-based MC simulations, which alters the dimensions of the simulation box while maintaining a constant volume (see Figure 1). The move involves scaling the length of

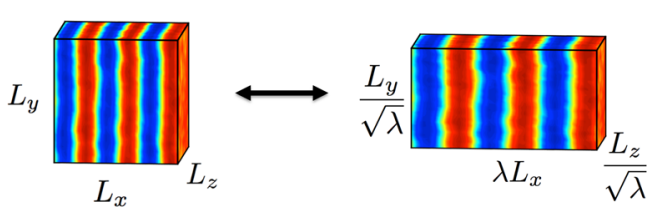

Figure 1. Illustration of the MC box move, which alters the lamellar period by modifying the dimensions of the simulation box while conserving the total volume, $V=L_{x} L_{y} L_{z}$.

the box in the direction normal to the lamellae (labeled $x$ ) by $\lambda$ $=\exp (\xi)$, where $\xi$ is a random number generated from a uniform distribution between $-\xi_{\max }$ and $\xi_{\max }$. To maintain the volume of the box, the remaining two directions ( $y$ and $z$ ) are scaled by $\lambda^{-1 / 2}$. During the start of the equilibration stage, $\xi_{\max }$ is adjusted to achieve an acceptance rate of $40 \%$.

In our implementation of the box move, we fix the number of grid points, $m$, in each direction, $\nu$, and just vary the grid spacings, $\Delta_{\nu}$. Thus, the move modifies $\chi_{\mathrm{e} 2}$ due to the changing cutoffs, $k_{\nu} \leq \pi L_{\nu} / m$, and therefore $\chi_{\mathrm{b}}$ needs to be adjusted in order to keep $\chi_{\mathrm{e} 2}$ constant during the simulation. Nevertheless, 


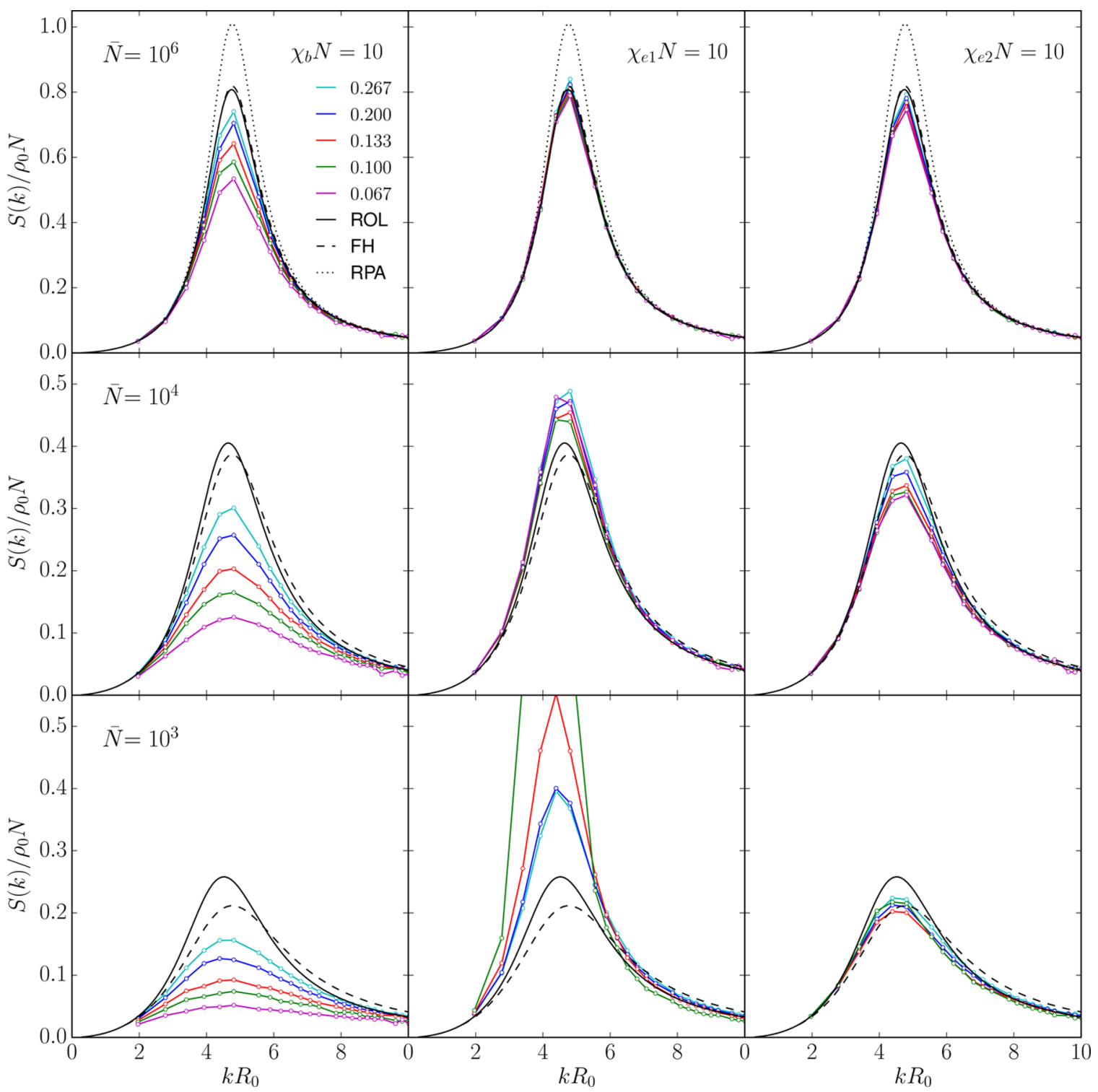

Figure 2. Structure function calculated for $\bar{N}=10^{6}$ (top row), $10^{4}$ (middle row), and $10^{3}$ (bottom row) at $\chi_{\mathrm{b}} N=10$ (left column), $\chi_{\mathrm{el}} N=10$ (middle column) and $\chi_{\mathrm{e} 2} N=10$ (right column) using various grid spacings $\Delta / R_{0}$ (colored curves). Predictions from $\mathrm{RPA}^{3} \mathrm{FH}^{7}$ and $\mathrm{ROL}^{12}(\mathrm{black}$ curves) are included for comparison. The different curves are specified by the legend in the top left panel.

the variation in $\chi_{\mathrm{b}}$ remains tiny provided the box stays relatively cubic, i.e., $L_{x} \approx L_{y}=L_{z}$. For $\bar{N}=10^{3}$ with $L=3.2 R_{0}$ and $m=24$, the change in $\chi_{\mathrm{b}}$ is less than $0.1 \%$ for a $5 \%$ change in $L_{x}$; for larger $\bar{N}$ or lower resolutions, the change is even smaller (e.g., less than $0.002 \%$ for $\bar{N}=10^{6}$ ). If the simulation box ever deviates too much from cubic, we use the result to improve our estimate of the lamellar period and redo the simulation with a more appropriately sized cubic box.

\section{RESULTS}

Structure Function. We begin our study by investigating the structure function in the disordered state ${ }^{25}$

$$
\begin{aligned}
\frac{S(\mathbf{k})}{\rho_{0} N} & =\frac{\rho_{\mathrm{c}}}{V}\left\langle\delta \hat{\phi}_{\mathrm{A}}(\mathbf{k}) \delta \hat{\phi}_{\mathrm{A}}(-\mathbf{k})\right\rangle \\
& =\frac{n}{\left(V \chi_{\mathrm{b}} N\right)^{2}}\left\langle W_{-}(\mathbf{k}) W_{-}(-\mathbf{k})\right\rangle-\frac{1}{2 \chi_{\mathrm{b}} N}
\end{aligned}
$$

which is routinely measured by small-angle scattering experiments to detect the presence of composition fluctuations. ${ }^{3}$ In the disorder state, the structure function is spherically symmetric [i.e., $S(\mathbf{k}) \rightarrow S(k)$ ] with a single broad peak at a wavevector, $k^{*}$, corresponding to the typical domain size and an amplitude, $S\left(k^{*}\right)$, related to the level of segregation among the A and B components. Our interest in $S(k)$ is twofold; naturally we wish to compare our simulations to the RPA, FH, and ROL predictions, but $S(k)$ is also an ideal quantity for examining the effects of the UV divergence. Figure 2 plots MC-FTS results for $\bar{N}=10^{6}, 10^{4}$, and $10^{3}$, obtained using a cubic simulation box of size $L=3.2 R_{0}$.

The first column of panels in Figure 2 shows $S(k)$ for the different $\bar{N}$ values at a fixed value of the bare interaction, $\chi_{\mathrm{b}} N=$ 10 , close to the ODT. Each plot includes data for a range of different mesh resolutions $\Delta / R_{0}$ (colored curves) along with theoretical predictions (black curves). The amplitude of the peak diminishes as the mesh resolution is increased due to the UV divergence. This is because the finer meshes permit shorter 
wavelength fluctuations, which in turn reduce the number of contacts between A and B segments (see eq 12), thus lowering the level of segregation. The effective $\chi_{\mathrm{e}}$ should ideally correct for this effect.

Indeed, ref 25 found that the curves for different resolutions collapse when the simulations are performed at a fixed $\chi_{\mathrm{e} 1} N=$ 10. Those results are repeated in the second column of Figure 2 , but with improved statistics, higher resolutions, and over a larger range of $\bar{N}$. As before, the collapse of the data is nearly perfect for $\bar{N}=10^{6}$. Furthermore, the master curve nicely agrees with the theoretical predictions of $\mathrm{FH}^{7}$ and $\mathrm{ROL},{ }^{12}$ which are nearly identical at this $\bar{N} .{ }^{34}$ For $\bar{N}=10^{4}$, there is still a reasonable collapse of the data, but not quite as good. Moreover, the simulation peak is about $25 \%$ higher than the theoretical predictions. For $\bar{N}=10^{3}$, however, the renormalization of the interaction parameter completely fails to control the UV divergence.

The last column of Figure 2 compares the performance of our new effective $\chi_{\mathrm{e} 2}$. The difference between $\chi_{\mathrm{e} 1}$ and $\chi_{\mathrm{e} 2}$ is relatively minor at $\bar{N}=10^{6}$, and so we retain a similar level of convergence. At $\bar{N}=10^{4}$, the collapse is slightly improved, keeping in mind that the focus is on the finer mesh resolutions. The truly stunning improvement, though, occurs for $\bar{N}=10^{3}$, where there is excellent collapse of the data for $\chi_{\mathrm{e} 2} N=10$ compared to complete failure for $\chi_{\mathrm{e} 1} N=10$. Admittedly, the quality of the collapse is aided somewhat by the fact that the approach to the master curve becomes nonmonotonic at this lower $\bar{N}$. In any case, the master curves compare well with the $\mathrm{FH}$ and ROL predictions over the full range of $\bar{N}$. Not only is there a similar reduction in the peak height, $S\left(k^{*}\right)$, with decreasing $\bar{N}$, there is also a slight shift in the peak position, $k^{*}$, toward smaller wavevectors consistent with ROL. Although this shift is ignored by the FH calculation, ${ }^{7}$ it does occur in the more refined versions of the theory. ${ }^{35,36}$

Although $\chi_{\mathrm{e} 1}$ and $\chi_{\mathrm{e} 2}$ collapse the data for sufficiently fine meshes, albeit with differing levels of success, the convergence nevertheless breaks down if the mesh becomes too fine, due to a divergence in $\chi_{\mathrm{b}}$ illustrated in Figure 3 . In the case of $\chi_{\mathrm{e} 1}$, the divergence is obvious from the form of eq 8 and corresponds to $\Delta / l \rightarrow 6 \alpha / \pi$. As the divergence is approached, $\chi_{\mathrm{b}} N$ becomes enormous, resulting in various numerical difficulties, which is why we do not provide results for $\Delta / R_{0}=0.067$ at $\bar{N}=10^{3}$.

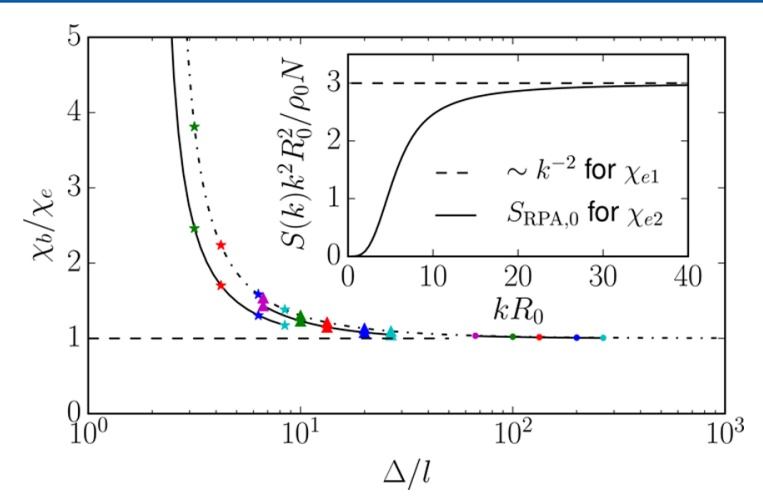

Figure 3. Ratios of the bare and effective interaction parameters for the different mesh resolutions, $\Delta / R_{0}$, in Figure 2. The symbols for $\bar{N}=$ $10^{3}(\star), 10^{4}(\triangle)$, and $10^{6}(\bullet)$ are plotted using the same color scheme as Figure 2 . The dash-dotted and solid curves correspond to eqs 8 and 13 for $\chi_{\mathrm{e} 1}$ and $\chi_{\mathrm{e} 2}$, respectively. The dashed and solid curves in the inset compare the integrands for $\int S(k) k^{2} \mathrm{~d} k$ used in the calculation of $\chi_{\mathrm{e} 1}$ and $\chi_{\mathrm{e} 2}$, respectively.
From our experience, $\chi_{\mathrm{e} 2}$ works well provided $\chi_{\mathrm{b}} / \chi_{\mathrm{e} 2} \lesssim 3$, while $\chi_{\mathrm{e} 1}$ falters at much smaller ratios of $\chi_{\mathrm{b}} / \chi_{\mathrm{e} 1}$.

Lamellar Period. Here we improve upon the MC-FTS of ref 25 , where the dimensions of the simulation box were fixed. In that study, the size, $L$, was chosen to fit a lamellar phase with a $\{211\}$ orientation (as specified by the usual Miller indices $\{h k l\})$, assuming the SCFT prediction for the period, $D_{\text {SCFT, }}$ evaluated at the effective $\chi_{\mathrm{e} 1} N$. Here, this assumption is used to initialize our system. In particular, we start with a $\{h 00\}$ lamellar phase in a cubic box of size $L=h D_{\text {SCFT }}$. This time, however, we allow the period to equilibrate by varying the dimensions of the simulation box, using the MC move illustrated in Figure 1.

The equilibrated period for $\bar{N}=10^{4}$ is plotted in Figure 4 from MC-FTS using various mesh resolutions and system sizes (colored curves) and compared to the SCFT prediction (dashed black curve). As was the case for $S(k)$, there is no consistency among the curves when plotted as a function of $\chi_{\mathrm{b}} N$. Again the UV divergence tends to reduce the segregation of the melt, and thus finer meshes result in shorter periods. For $\bar{N} \gtrsim 10^{4}$, the problem is rectified by plotting the results in terms of either $\chi_{\mathrm{e} 1} N$ or $\chi_{\mathrm{e} 2} N$. At the smaller $\bar{N}=10^{3}$, however, the curves no longer collapse when plotted as a function of $\chi_{\mathrm{e} 1} N$ (the spread among the curves is $\sim 3 \%$ ), but they still collapse for $\chi_{\mathrm{e} 2} N$ (see the bottom panel of Figure 5).

Remarkably, the MC-FTS prediction for the lamellar period agrees accurately with SCFT, provided that it is expressed as a function of $\chi_{\mathrm{e} 2} N$. In fact, the result for $\bar{N}=10^{8}$ (not shown) is the same as the SCFT prediction to within $0.1 \%$. Although the agreement becomes less with increasing fluctuations, as illustrated in Figure 5, the difference nevertheless remains within about $1 \%$ at our lowest $\bar{N}=10^{3}$. Interestingly, similar consistency with SCFT was recently reported for particle-based simulations. $^{27}$

Order-Disorder Transition (ODT). Our scans of the lamellar period in the previous section extend to the point where the lamellar phase disorders, thereby providing us with estimates of $\chi N$ and $D$ at the ODT. We now perform further simulations in order to refine these initial estimates of $(\chi N)_{\text {ODT }}$ and $D_{\mathrm{ODT}}$. To ensure confidence in our results, the simulations are done over a range of system sizes (i.e., different numbers of periods, $h)$ to assess finite-size effects.

To help cope with the first-order ODT, we use parallel tempering, ${ }^{25}$ which involves a series of simulations conducted in parallel at closely spaced values of $\chi_{\mathrm{e} 2} N$ spanning the ODT. In addition to the usual MC moves in each replica of the system, the method also exchanges replicas between neighboring values of $\chi_{\mathrm{e} 2} N$. Here the box move is omitted because of the disordered configurations, and so all the replicas are assigned a common cubic simulation box of fixed size, $L=h D_{\mathrm{ODT}}$, corresponding to our estimate of $D_{\mathrm{ODT}}$. Furthermore, the grid resolution is fixed at $m=8 h$. The ODT is then detected by a jump in the order parameter $\langle\Psi\rangle$, defined as the ensemble average of ${ }^{25}$

$$
\Psi=V^{-2} \max _{\mathbf{k}}\left[W_{-}(\mathbf{k}) W_{-}(-\mathbf{k})\right]
$$

In order to assess the nonequilibrium effects in our simulation, two parallel-tempering runs are performed for each ODT measurement: one where all the replicas start from a disorder configuration and another where they all begin from a lamellar configuration with $h$ lamellae. Both initial configurations are first equilibrated, at low and high values of $\chi_{\mathrm{e} 2} N$. The two 

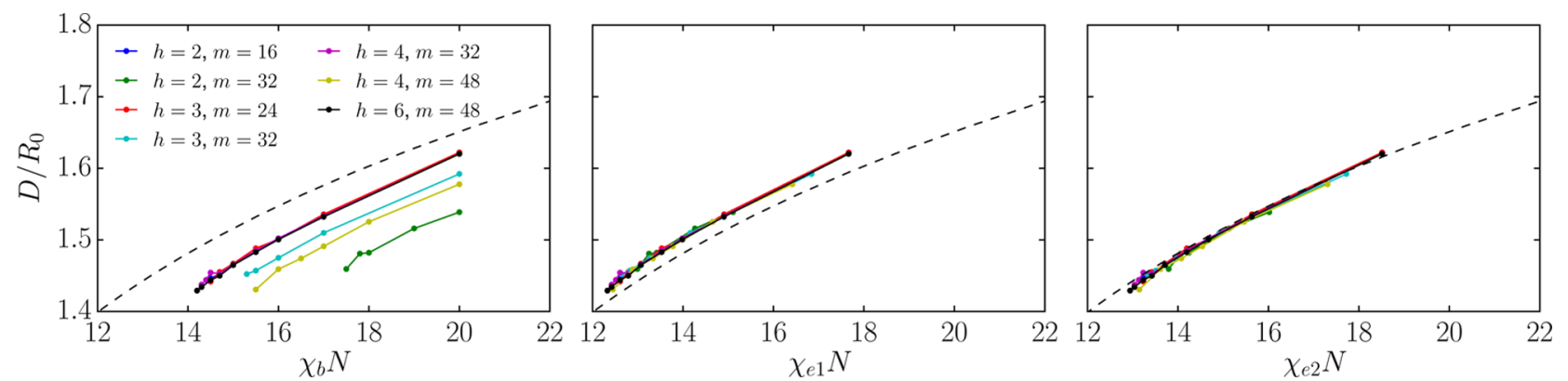

Figure 4. Lamellar period vs $\chi_{\mathrm{b}} N$ (left), $\chi_{\mathrm{e} 1} N$ (middle), and $\chi_{\mathrm{e} 2} N$ (right) calculated for $\bar{N}=10^{4}$ with various numbers of periods, $h$, and grid points, $\mathrm{m}^{3}$. The dashed curves denote the SCFT prediction.
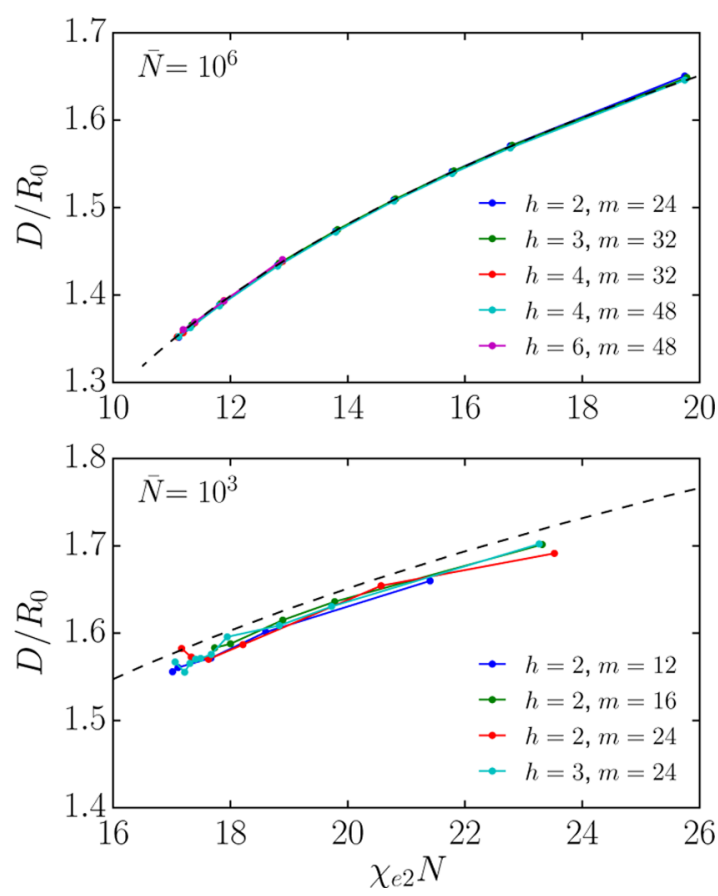

Figure 5. Lamellar period vs $\chi_{\mathrm{e} 2} N$ for $\bar{N}=10^{6}$ (top) and $\bar{N}=10^{3}$ (bottom) with various numbers of periods, $h$, and grid points, $m^{3}$. The dashed curve denotes the SCFT prediction.

parallel-tempering runs produce jumps in the order parameter below and above the true ODT, thereby bracketing $\left(\chi_{e 2} N\right)_{\mathrm{ODT}}$ by a metastability interval that narrows as equilibrium is approached. Unlike in particle-based simulations where the dynamics is generally slower at larger $\bar{N}$, the opposite is true of MC-FTS. For a particle-based model, an increase in $\bar{N}$ requires more molecules. However, the molecular coordinates are integrated out of the effective Hamiltonian (eq 6) for the MC-FTS, which then allows the system to equilibrate more quickly at larger $\bar{N}$ on account of the weaker composition fluctuations and the smaller discontinuity in the ODT.

Figure 6 displays our results from the parallel-tempering runs over a range of $\bar{N}$ values. For the weak first-order transition at $\bar{N}=10^{8}$, we are able to simulate systems containing up to $h=6$ compete lamellar periods. Although $h=2$ is too small to produce a sudden jump in $\langle\Psi\rangle$, a well-defined transition is evident in each of the larger boxes. For the largest $h=6$ box, the metastability interval between the jumps in $\langle\Psi\rangle$ from the disordered and ordered starts has not completely closed, but nevertheless it is narrow enough to provide an accurate estimate of $\left(\chi_{\mathrm{e} 2} N\right)_{\mathrm{ODT}}$. Interestingly, $\left(\chi_{\mathrm{e} 2} N\right)_{\mathrm{ODT}}$ does not
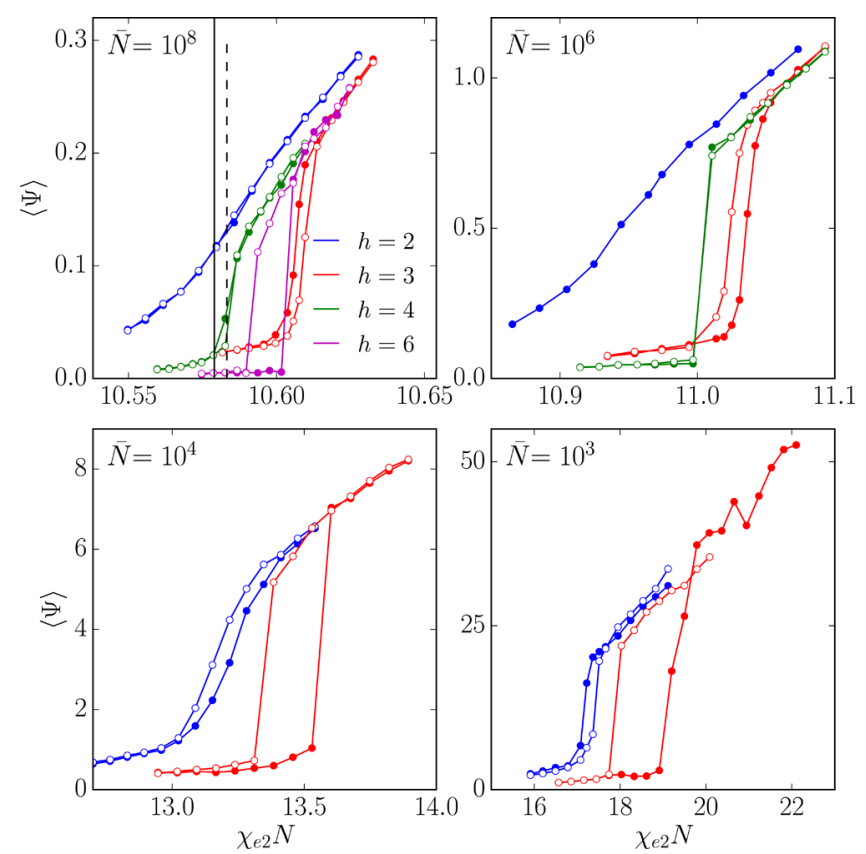

Figure 6. Order parameter vs $\chi_{\mathrm{e} 2} N$ for various values of $\bar{N}$. Solid and open symbols denote simulations started from disordered and lamellar configurations, respectively, in simulation boxes fitting $h=2$ (blue), 3 (red), 4 (green), and 6 (purple) lamellar periods. The vertical dashed and solid lines for $\bar{N}=10^{8}$ denote the FH prediction in eq 1 and the improved prediction from ref 9 , respectively.

converge to the thermodynamic limit monotonically as $h \rightarrow$ $\infty$. This may be a result of competing effects, such as the suppression of fluctuations due to the finite-size box versus the relief of frustration in larger boxes, which would presumably increase and decrease $\left(\chi_{\mathrm{e} 2} N\right)_{\mathrm{ODT}}$, respectively.

The first-order nature of the ODT becomes stronger with decreasing $\bar{N} .^{27}$ As as result, sudden jumps in $\langle\Psi\rangle$ eventually occur even for the smallest $h=2$ simulation boxes. The metastability intervals also become more persistent, which limits the size of boxes that can be considered. Nevertheless, the shifts due to finite-size effects and the metastability intervals due to nonequilibrium effects remain sufficiently small that we can still obtain good estimates for $\left(\chi_{\mathrm{e} 2} N\right)_{\mathrm{ODT}}$.

The resulting fluctuation correction to the ODT, $\left(\chi_{\mathrm{e}} N\right)_{\mathrm{ODT}}-$ 10.495 , is plotted in Figure 7 for both $\chi_{\mathrm{e} 1}$ and $\chi_{\mathrm{e} 2}$ with downward- and upward-pointing triangles, respectively. The values are obtained from our largest system sizes with error bars corresponding to the width of the metastability interval. Also included is the result for an earlier Langevin FTS that used the same saddle-point approximation for the pressure field; ${ }^{24}$ the 


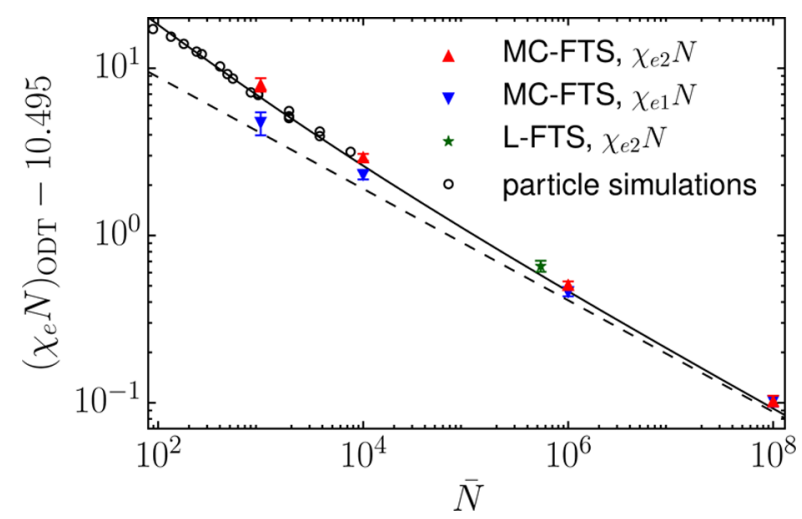

Figure 7. Shift in the ODT relative to mean-field theory evaluated using $\chi_{\mathrm{e} 1}$ from eq 8 (blue triangles) and $\chi_{\mathrm{e} 2}$ from eq 13 (red triangles). Also included are the ODTs from a Langevin FTS $^{24}$ (green star) and particle simulations ${ }^{18}$ (black circles). The dashed and solid curves denote the $\mathrm{FH}$ prediction in eq 1 and the empirical fit in eq 2, respectively.

data point (green star) is obtained by converting the $\left(\chi_{\mathrm{b}} N\right)_{\mathrm{ODT}}$ for their largest simulation box to $\left(\chi_{\mathrm{e}_{2}} N\right)_{\mathrm{ODT}}$. All the FTS results, including the one from ref 24 , agree remarkably well with the particle-based simulations (open circles) corresponding to the empirical fit in eq 2 (solid curve), provided we use $\chi_{\mathrm{e} 2} N$. We also compared the FTS results to $(\chi N)_{\mathrm{ODT}}=10.495$ $+39.0 \bar{N}^{-1 / 3}+106.5 \bar{N}^{-0.52}$, obtained by refitting the particle simulations assuming the coefficient of 39.0 derived by Mayes and Olvera de la Cruz. ${ }^{9}$ However, the fluctuation correction predicted by this fit differs from that of eq 2 by less than $4 \%$ over the range $\bar{N}=10^{2}-10^{8}$, and thus the agreement remains very similar.

Given our refined values for $\left(\chi_{\mathrm{e}} N\right)_{\mathrm{ODT}}$, the estimate of $D_{\mathrm{ODT}}$ can be improved by referring back to our simulation results with the box move. The improved values are plotted in Figure 8. Again, there is excellent agreement with particle-based

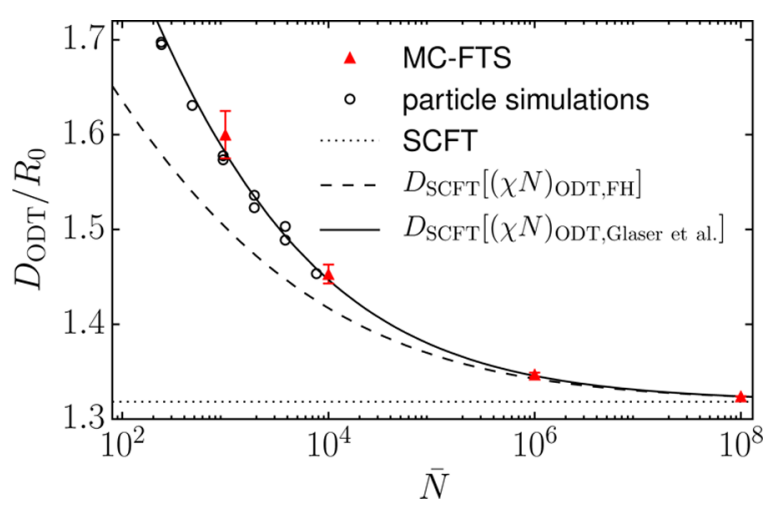

Figure 8. Lamellar period at the ODT, which is a quantity that does not depend on the definition of $\chi_{\mathrm{e}}$. Also included are results from particle simulations (open circles). ${ }^{27}$ The dashed and solid curves denote the SCFT period, $D_{\mathrm{SCFT}}$, evaluated at the $\mathrm{FH}$ prediction in eq 1 and the empirical fit in eq 2 , respectively.

simulations (open symbols). Furthermore, our MC-FTS results (triangular symbols) nicely match the SCFT prediction (solid curve) evaluated at the empirical estimate in eq 2 . Note that the lamellar period at the ODT is uniquely defined, and thus this agreement does not depend on the definition of $\chi_{\mathrm{e}}$.
The initial estimates of $D_{\text {ODT }}$ used for the parallel-tempering runs differ from the improved values in Figure 8 by less than $1 \%$. An inaccuracy in $D$ would tend to cause an upward shift in $\left(\chi_{\mathrm{e}} N\right)_{\text {ODT }}$ due to frustration effects associated with the incommensurability. ${ }^{27,37-41}$ However, from experience, a $1 \%$ inaccuracy is too small to have a significant effect on the ODT. Our confidence that the commensurability was sufficiently accurate is further bolstered by the fact that the paralleltempering runs from the disordered state spontaneously ordered into lamellae with the expected $\{h 00\}$ orientations. The only real exception was for $h=3$, where $\{221\}$ orientations were frequently observed, but this is because they possess the exact same domain spacing as the $\{300\}$ ones. $^{27}$

Compression Modulus and Lamellar Profile. A convenient byproduct of the MC box move is the smectic (or layer) compression modulus $B$ of the lamellae. It is obtained by simply monitoring fluctuations in $L_{x}$, the box size normal to the lamellae (see Figure 1). Assuming small fluctuations, the free energy cost

$$
\delta F=\frac{1}{2} B V \epsilon^{2}
$$

varies quadratically with the relative deviation from equilibrium, $\epsilon=L_{x} /\left\langle L_{x}\right\rangle-1$. Thus, it immediately follows from the equipartition theorem, $\langle\delta F\rangle={ }^{1} /{ }_{2} k_{\mathrm{B}} T$, that

$$
\bar{B} \equiv \frac{B}{\rho_{\mathrm{c}} k_{\mathrm{B}} T}=\frac{1}{n\left\langle\epsilon^{2}\right\rangle}
$$

The dimensionless modulus $\bar{B}$ is plotted as a function of $\chi_{\mathrm{e} 2} \mathrm{~N}$ in the first column of Figure 9 for $\bar{N}=10^{4}$ and $10^{6}$. The different curves for various system sizes overlap remarkably well, indicating that the finite-size effects for this quantity are minimal. For $\chi_{\mathrm{e} 2} N \gg\left(\chi_{\mathrm{e} 2} N\right)_{\mathrm{ODT}}$, we find good agreement with SCFT (black curve), suggesting that $\bar{B}$ is relatively unaffected by fluctuations. However, close to the ODT, there is a significant reduction in $\bar{B}$ relative to SCFT, confirming similar findings from recent particle-based simulation. ${ }^{27}$ Notice that for small $\bar{N}$ we can measure $\bar{B}$ well beyond the ODT because the metastable lamellae are relatively long-lived due to the firstorder transition. ${ }^{42}$ For large $\bar{N}$, on the other hand, the lamellar configurations will occasionally disorder even on the orderedside of the ODT (albeit momentarily), which limits how close we can approach the ODT.

Using an approach similar to the FH theory, ${ }^{7,8}$ Amundson and Helfand ${ }^{43}$ predicted that the smectic compressibility near the ODT is related to the amplitude of the composition profile by

$$
\bar{B}=x^{* 2} \tilde{F}^{\prime \prime}\left(x^{*}\right) \phi_{\mathrm{A}, 1}{ }^{2}=13.7878 \phi_{\mathrm{A}, 1}^{2}
$$

where $\tilde{F}(x)=N \rho_{0} / S_{\mathrm{RPA}}(k), x=k^{2} R_{0}^{2} / 6$, and $x^{*}=3.7852$. In their weak-segregation calculation, the amplitude of the composition profile is given by the first harmonic. In principle, it is evaluated by $\phi_{\mathrm{A}, 1}=2 \mathrm{~L}\left\langle\delta \hat{\phi}_{\mathrm{A}}\left(\mathrm{k}^{*}\right)\right\rangle \mathrm{l} / \mathrm{V}$, but in finite systems, we have to account for translational drift of the lamellae. Rather than correcting for this by continuously shifting the profile, ${ }^{27}$ we evaluate the amplitude using $\phi_{\mathrm{A}, 1}=2\left\langle\left|\delta \hat{\phi}_{\mathrm{A}}\left(\mathbf{k}^{*}\right)\right|\right\rangle / V$. As expected, the difference between the two expressions for $\phi_{\mathrm{A}, 1}$ diminishes as the system size increases, particularly when $\chi_{\mathrm{e} 2} N$ $\gg\left(\chi_{\mathrm{e} 2} N\right)_{\mathrm{ODT}}$.

The dependence of $\phi_{\mathrm{A}, 1}$ on $\chi_{\mathrm{e} 2} N$ is displayed in the second column of Figure 9. Again the data collapse well for the different grid resolutions and system sizes. Given the 

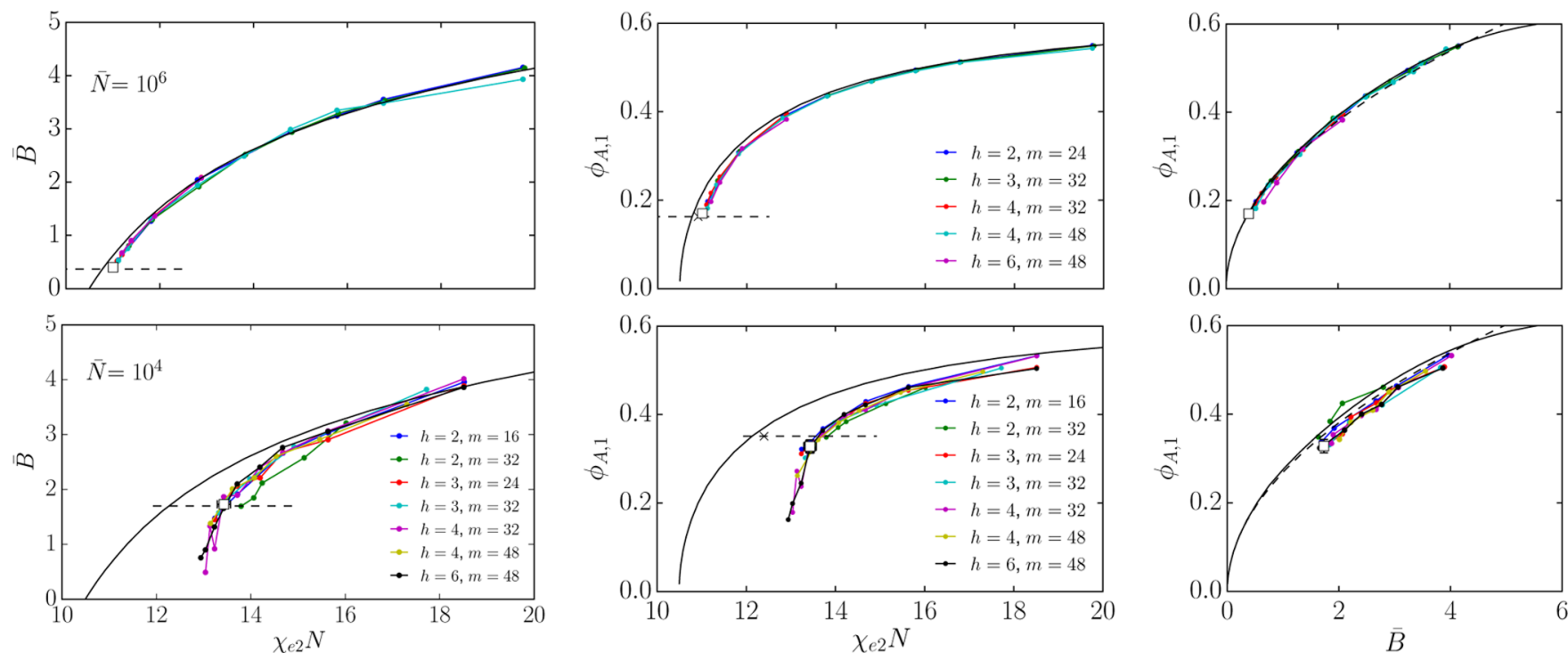

Figure 9. Compression modulus $\bar{B}$ vs $\chi_{\mathrm{e} 2} N$ (left), amplitude of the composition profile $\phi_{\mathrm{A}, 1}$ vs $\chi_{\mathrm{e} 2} N$ (middle), and $\phi_{\mathrm{A}, 1}$ vs $\bar{B}$ (right) obtained from simulation boxes with various numbers of lamellar periods, $h$, and grid points, $m^{3}$. Results are provide for $\bar{N}=10^{6}$ (upper panels) and $\bar{N}=10^{4}$ (lower panels). Open squares indicate ODT values, and solid curves denote SCFT predictions. The dashed lines correspond to eq 21 (left), eq 20 (middle), and eq 19 (right).

relationship between $\bar{B}$ and $\phi_{\mathrm{A}, 1}$ (eq 19), it is not surprising that there is a drop in $\phi_{\mathrm{A}, 1}$ with respect to SCFT (solid curve) as the ODT is approached. The quadratic relationship between $\bar{B}$ and $\phi_{\mathrm{A}, 1}$ is tested in the third column of Figure 9. For $\bar{N}=10^{6}$, we find excellent agreement with eq 19 (dashed curve) as well as with SCFT (solid curve). For $\bar{N}=10^{4}$, both predictions slightly overestimate $\phi_{\mathrm{A}, 1}$ for a given $\bar{B}$, but nevertheless the agreement is still rather good.

According to $\mathrm{FH}$ theory, ${ }^{7}$ the amplitude of the composition profile at the ODT varies with $\bar{N}$ as

$$
\phi_{\mathrm{A}, 1, \mathrm{ODT}}=1.6294 \bar{N}^{-1 / 6}
$$

which in turn implies that

$$
\bar{B}_{\mathrm{ODT}}=36.609 \bar{N}^{-1 / 3}
$$

These predictions are denoted by the horizontal dashed lines in Figure 9, and indeed they correspond well with the values of $\phi_{\mathrm{A}, 1}$ and $\bar{B}$ at the ODT (square symbols). Hence, their deviation away from the SCFT prediction can be attributed to fluctuation effects, which are accurately accounted for by the FH treatment provided $\bar{N}$ is sufficiently large.

Naturally, the $\mathrm{FH}$ predictions for $\phi_{\mathrm{A}, 1, \mathrm{ODT}}$ and $\bar{B}_{\mathrm{ODT}}$ break down for small values of $\bar{N}$ as illustrated in Figure 10. Interestingly, the significant deviations from eqs 20 and 21 observed for $\bar{N}=10^{3}$ are in good agreement with the particlebased simulations in ref 37 (circular symbols). Of course, the deviation of $\phi_{\mathrm{A}, 1, \mathrm{ODT}}$ from eq 20 is necessitated by the fact the composition profile has to remain between 0 and 1 . The solid curve in the upper panel of Figure 10 shows a fit, $\phi_{\mathrm{A}, 1, \mathrm{ODT}}=$ $\left(\left(1.63 \bar{N}^{-1 / 6}\right)^{-\alpha}+\phi_{0}^{-\alpha}\right)^{-1 / \alpha}$, to the MC-FTS and particle simulation data with fitting parameters of $\phi_{0}=0.442$ and $\alpha=$ 4.35, which gives a physically reasonable limit for $\bar{N} \rightarrow 0$.

\section{DISCUSSION}

This study succeeded, for the first time, in applying 3D fieldtheoretic simulation (FTS) to diblock copolymer melts at an invariant polymerization index well within the experimental regime. The biggest challenge to overcome was the ultraviolet
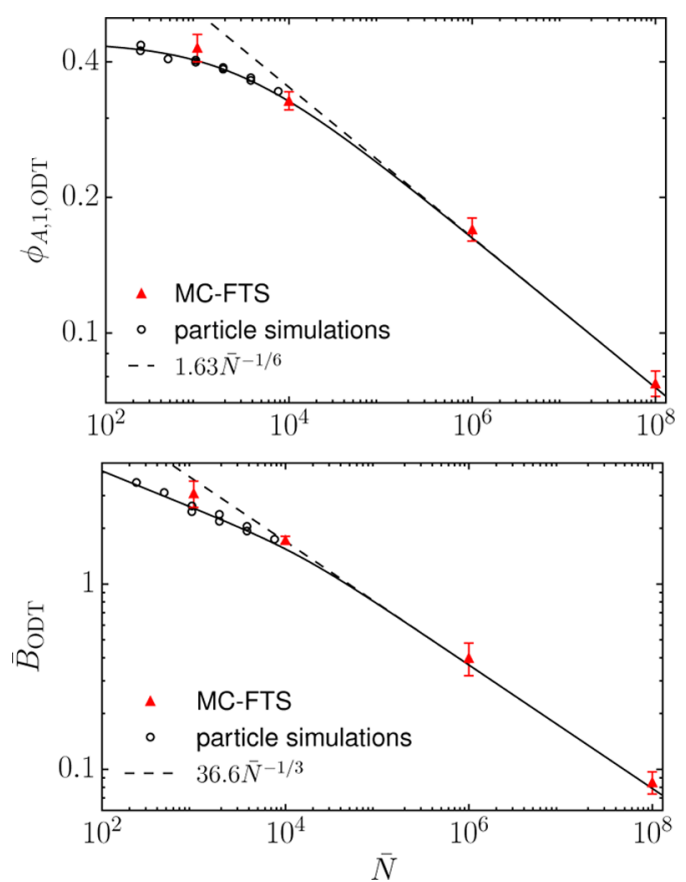

Figure 10. Amplitude of the composition profile $\phi_{\mathrm{A}, 1, \mathrm{ODT}}$ (top) and compression modulus $\bar{B}_{\mathrm{ODT}}$ (bottom) at the ODT. Also included are results from particle simulations (open circles). ${ }^{27}$ Dashed lines denote the $\mathrm{FH}$ predictions from eqs 20 and 21 , and solid curves are simple fits to guide the eye.

(UV) divergence, which tends to disorder the melt when the grid spacing is reduced. This was previously ${ }^{25}$ dealt with by using an effective Flory-Huggins interaction parameter, $\chi_{\mathrm{e} 1}$, introduced by Olvera de la Cruz et al. ${ }^{26}$ Unfortunately, it fails to control the stronger UV divergence that occurs when $\bar{N} \lesssim 10^{4}$. To overcome this problem, we derived an alternative interaction parameter, $\chi_{\mathrm{e} 2}$, following an approach analogous to that of Müller and Binder. ${ }^{30}$ The two definitions of $\chi_{\mathrm{e}}$ were tested for a range of $\bar{N}$ by evaluating the disordered-state 
structure function, $S(k)$, at different grid resolutions. The new $\chi_{\mathrm{e} 2}$ successfully collapses the data down to $\bar{N}=10^{3}$. The use of $\chi_{\mathrm{e} 2}$ also leads to good agreement with SCFT for the period and smectic compression modulus of well-ordered lamellae, consistent with recent particle-based simulations. ${ }^{27}$ Furthermore, it results in good agreement with the empirical eq 2 for the ODT. ${ }^{18}$

The difference between the two interaction parameters is that $\chi_{\mathrm{e} 2}$ in eq 13 involves an integral over the RPA structure function at $\chi=0, S_{\mathrm{RPA}, 0}(k)$, while $\chi_{\mathrm{e} 1}$ in eq 8 uses its asymptotic $k^{-2}$ behavior, eq 14 . This approximation of $S_{\mathrm{RPA}, 0}(k)$ adds a contribution to $\chi_{\mathrm{el}}$ from long-wavelength fluctuations that would appear to the molecules as a uniform shift in the field, which thus should not affect their statistics. Integrating instead over the full $S_{\mathrm{RPA}, 0}(k)$ cuts off this contribution from wavelengths larger than the molecules (see the inset of Figure 3 ). However, in doing so, $\chi_{\mathrm{e} 2}$ becomes somewhat dependent on the molecular architecture and, in particular, on the invariant degree of polymerization, as is the case for the effective interaction parameter defined by Müller and Binder. ${ }^{30}$ This is because the cutoff is $\bar{N}$ dependent (i.e., $k \lesssim 2 \pi / R_{0}=2 \pi / l \bar{N}^{1 / 2}$ ). The dependence of $\chi_{\mathrm{e} 2}$ on $\bar{N}$ is somewhat unappealing, but the improvement over $\chi_{\mathrm{e} 1}$ is undeniable. A way to avoid the $\bar{N}$ dependence might be to calculate $\chi_{\mathrm{e}}$ by extending the loop expansion of ROL to higher order; ${ }^{12}$ for consistency, the expansion should be done with the same saddle-point approximation for the pressure field used in MC-FTS. Another alternative is to determine $\chi_{\mathrm{e}}$ from fitting the peak of the structure function to ROL theory, as was done for the particlebased simulations. $^{18,27}$ However, such a calibration would be tedious, especially considering that any change in the reduced mesh size, $\Delta / l$, would require a recalibration of $\chi_{\mathrm{e}}$. Nevetheless, at some point, it would be good to try this for at least one ratio of $\Delta / l$, although we would not expect much of a change given that the current $\chi_{\mathrm{e} 2}$ already produces good agreement with the ROL structure function.

MC-FTS does involve one approximation to the statistical mechanics of the standard Gaussian-chain model; it evaluates the pressure field using the saddle-point approximation. This mean-field treatment of incompressibility seems completely reasonable, and indeed an earlier $2 \mathrm{D}$ study in ref 21 found almost perfect agreement with the full CL-FTS, which includes fluctuations of the pressure field. Thus, it is puzzling that a more recent 3D CL-FTS by Lennon et al. ${ }^{22}$ for $\bar{N}=5.4 \times 10^{5}$ reported a fluctuation correction to the ODT that is about twice the size of ours. Although this might suggest an inaccuracy with the saddle-point approximation, we suspect that the issue lies with the CL-FTS prediction, given that it also disagrees with the empirical eq 2 from the particle simulations. There could potentially be a problem with the complex Langevin method, ${ }^{19,44}$ which is not as well grounded as the conventional Langevin simulation, but it may also just be related to the UV divergence. An increase in dimensionality should improve the accuracy of the saddle-point approximation, whereas it amplifies the UV divergence from what should be a weak logarithmic divergence in $2 \mathrm{D}$. On top of that the strict enforcement of incompressibility will change the nature of the UV divergence, ${ }^{45}$ and so we should not necessarily expect $\chi_{\mathrm{e} 1}$ (or $\chi_{\mathrm{e} 2}$ ) to compensate for the UV divergence in the CL-FTS. In any case, more recent CL-FTS have modified the model by smearing the interactions, introducing a finite compressibility and switching to a discrete polymer model in order to avoid the UV divergence; ${ }^{46}$ this has also permitted the CL-FTS to handle diblocks of a lower $\bar{N}=10^{5}$, although this is still well above the experimental regime.

The similarity of phase behavior observed in experiment among chemically different diblock copolymer systems ${ }^{10}$ has long suggested an underlying universality, whereby all systems approach the mean-field predictions of the standard Gaussianchain model $^{4}$ with its minimal set of parameters (i.e., $\chi N, f$, and $\left.a_{\mathrm{A}} / a_{\mathrm{B}}\right)$ in the limit of infinite molecular weight. Furthermore, it is believed that $\bar{N}$ is the dominant parameter that controls the fluctuation effects causing deviations from mean-field theory for finite molecular weights. A string of detailed studies by Morse and co-workers ${ }^{17,18,27,47}$ have put this hypothesis on a strong footing in recent years. By dealing with the UV divergence of the standard model, our results are expressed in terms of its parameters. If we instead just changed the model to avoid the UV divergence, the interaction parameter (e.g., $\chi_{\mathrm{b}}$ ) would no longer correspond to that of the standard model, as is the usual case for particle-based simulations. This is fine, but then the interaction parameter would still have to be appropriately calibrated and validated in order to make contact with the universal behavior, such as the ROL predictions for $S(k)$ or eq 2 for the ODT. The mapping of simulations onto the universal behavior of the standard model cannot be overstated. Not only does it permit direct quantitative comparisons among the different simulation models, it also opens up the possibility for quantitative comparisons with experiment. Indeed, Gillard et al. ${ }^{48}$ just recently compared an experimental ODT to eq 2 by determining $\chi_{\mathrm{e}}$ from a fit to the ROL structure function. The resulting agreement was quite reasonable, and it is entirely possible that the modest discrepancy can be largely attributed to the polydispersity (PDI $=1.1$ ) of the experimental molecules.

\section{CONCLUSIONS}

Detailed field-theoretic simulations were performed on the standard Gaussian-chain model for melts of lamellar-forming diblock copolymer, using a Monte Carlo variant (MC-FTS) where incompressibility is enforced within the mean-field approximation. Our key achievement was the introduction of a new effective interaction parameter, $\chi_{\mathrm{e} 2}$, capable of controlling its UV divergence down to invariant polymerization indices of $\bar{N} \approx 10^{3}$, thereby allowing MC-FTS to handle realistic experimental conditions. The simulations also benefitted from the use of GPUs, which allowed for large simulation boxes containing up to six lamellar periods. Finite-size effects where further alleviated by an efficient MC box move that permitted the lamellae to adjust their period.

With these new improvements, we were able to locate the order-disorder transition (ODT) with greater accuracy over a wider range of invariant polymerizations (i.e., $\bar{N}=10^{3}-10^{8}$ ). This now reveals better convergence to the $\mathrm{FH}$ prediction (eq 1 ) in the large- $\bar{N}$ limit but also confirms significant deviations from the $\mathrm{FH}$ theory in the experimental regime, consistent with the empirical eq 2 from particle-based simulations. Our study also finds that the period and smectic compressibility of wellordered lamellae agree with SCFT predictions, which again corroborates a conclusion reached by the particle simulations. Furthermore, we observe a similar dip in the compressibility relative to SCFT as the ODT is approached. Here, however, we are able to attribute this dip, and an analogous one for the amplitude of the composition profile, to fluctuation effects.

In light of this success, MC-FTS now becomes a viable alternative to conventional particle-based simulation of block 
copolymers. Naturally, it will be particularly suited to highmolecular-weight polymers, but the greatest advantage of MCFTS will undoubtedly be that it possesses the versatility of SCFT. While the computational cost of particle simulation increases dramatically for complex architectures and for multicomponent blends, such generalizations will be relatively straightforward for MC-FTS.

\section{AUTHOR INFORMATION}

\section{Corresponding Authors}

*E-mail bvorselaars@lincoln.ac.uk (B.V.).

*E-mail mwmatsen@uwaterloo.ca (M.W.M.).

\section{Present Address}

B.V.: School of Mathematics and Physics, University of Lincoln, Brayford Pool, Lincoln LN6 7TS, U.K.

\section{Notes}

The authors declare no competing financial interest.

\section{ACKNOWLEDGMENTS}

We thank Frank Bates and David Morse for useful discussions and Jian Qin for providing a computer program for $S_{\mathrm{ROL}}(k)$. This work was funded by NSF under the Center for Sustainable Polymers (CHE-1413862), and computer resources were provided by SHARCNET.

\section{REFERENCES}

(1) (a) Stupp, S. I.; LeBonheur, V.; Walker, K.; Li, L. S.; Huggins, K. E.; Keser, M.; Amstutz, A. Science 1997, 276, 384-389. (b) Bates, F. S.; Hillmyer, M. A.; Lodge, T. P.; Bates, C. M.; Delaney, K. T.; Fredrickson, G. H. Science 2012, 336, 434-440.

(2) (a) Bates, F. S.; Fredrickson, G. Phys. Today 1999, 52, 32-38. (b) Hadjichristidis, N.; Pispas, S.; Floudas, G. Block Copolymers: Synthetic Strategies, Physical Properties, and Applications; John Wiley \& Sons, Inc.: 2003. (c) Segalman, R. A. Mater. Sci. Eng., R 2005, 48, 191226.

(3) Leibler, L. Macromolecules 1980, 13, 1602-1617.

(4) Matsen, M. W. J. Phys.: Condens. Matter 2002, 14, R21-R47.

(5) Fredrickson, G. H. The Equilibrium Theory of Inhomogeneous Polymers; Clarendon Press: Oxford, 2006.

(6) Edwards, S. F. Proc. Phys. Soc., London 1965, 85, 613-624.

(7) Fredrickson, G. H.; Helfand, E. J. Chem. Phys. 1987, 87, 697-705.

(8) Brazovskii, S. A. Sov. Phys.-JETP 1975, 41, 85-89.

(9) Mayes, A. M.; de la Cruz, M. O. J. Chem. Phys. 1991, 95, 46704677.

(10) Bates, F. S.; Schulz, M. F.; Khandpur, A. K.; Forster, S.; Rosedale, J. H.; Almdal, K.; Mortensen, K. Faraday Discuss. 1994, 98, $7-18$.

(11) Morse, D. C.; Chung, J. K. J. Chem. Phys. 2009, 130, 224901.

(12) Qin, J.; Grzywacz, P.; Morse, D. C. J. Chem. Phys. 2011, 135, 084902 .

(13) Morse, D. C.; Qin, J. J. Chem. Phys. 2011, 134, 084902.

(14) (a) Yamakawa, H. Modern Theory of Polymer Solutions; Harperas Chemistry Series; Harper \& Row: 1971. (b) Müller, M.; Binder, K.; Schäfer, L. Macromolecules 2000, 33, 4568-4580. (c) Wang, Z.-G. J. Chem. Phys. 2002, 117, 481-500. (d) Wittmer, J. P.; Meyer, H.; Baschnagel, J.; Johner, A.; Obukhov, S.; Mattioni, L.; Müller, M.; Semenov, A. N. Phys. Rev. Lett. 2004, 93, 147801. (e) Beckrich, P.; Johner, A.; Semenov, A. N.; Obukhov, S. P.; Benoit, H.; Wittmer, J. P. Macromolecules 2007, 40, 3805-3814.

(15) Allen, M. P.; Tildesley, D. J. Computer Simulation of Liquids; Clarendon Press: New York, 1989.

(16) Frenkel, D.; Smit, B. Understanding Molecular Simulation: From Algorithms to Applications, 2nd ed.; Academic Press: 2002.

(17) Glaser, J.; Qin, J.; Medapuram, P.; Morse, D. C. Macromolecules 2014, 47, 851-869.
(18) Glaser, J.; Medapuram, P.; Beardsley, T. M.; Matsen, M. W.; Morse, D. C. Phys. Rev. Lett. 2014, 113, 068302.

(19) Fredrickson, G. H.; Ganesan, V.; Drolet, F. Macromolecules 2002, 35, 16-39.

(20) Parisi, G. Phys. Lett. B 1983, 131, 393-395.

(21) Düchs, D.; Ganesan, V.; Fredrickson, G. H.; Schmid, F. Macromolecules 2003, 36, 9237-9248.

(22) Lennon, E. M.; Katsov, K.; Fredrickson, G. H. Phys. Rev. Lett. 2008, 101, 138302.

(23) Düchs, D.; Schmid, F. J. Chem. Phys. 2004, 121, 2798-2805.

(24) Alexander-Katz, A.; Fredrickson, G. H. Macromolecules 2007, 40, 4075-4087.

(25) Stasiak, P.; Matsen, M. W. Macromolecules 2013, 46, 80378045.

(26) Olvera de la Cruz, M.; Edwards, S. F.; Sanchez, I. C. J. Chem. Phys. 1988, 89, 1704-1708.

(27) Medapuram, P.; Glaser, J.; Morse, D. C. Macromolecules 2015, $48,819-839$.

(28) Müller, M.; Schmid, F. In Advanced Computer Simulation Approaches for Soft Matter Sciences II; Holm, C., Kremer, K., Eds.; Advances in Polymer Science; Springer: Berlin, 2005; Vol. 185, pp 158.

(29) Delaney, K. T.; Fredrickson, G. H. Comput. Phys. Commun. 2013, 184, 2102-2110.

(30) Müller, M.; Binder, K. Macromolecules 1995, 28, 1825-1834.

(31) de Gennes, P. Scaling Concepts in Polymer Physics; Cornell University Press: 1979.

(32) Flory, P. J. Principles of Polymer Chemistry; Cornell University Press: 1953.

(33) Zong, J.; Wang, Q. J. Chem. Phys. 2013, 139, 124907.

(34) In ref 25 , there was a small mistake in the ROL curve for $\bar{N}=$ $10^{6}$; the corrected curve now agrees much better with the MC-FTS.

(35) Barrat, J.-L.; Fredrickson, G. H. J. Chem. Phys. 1991, 95, 12811289.

(36) Olvera de la Cruz, M. Phys. Rev. Lett. 1991, 67, 85-88.

(37) Luckhurst, G. R.; Stephens, R. A.; Phippen, R. W. Liq. Cryst. 1990, 8, 451-464.

(38) Wang, Q.; Yan, Q.; Nealey, P. F.; de Pablo, J. J. J. Chem. Phys. 2000, 112, 450-464.

(39) Martínez-Veracoechea, F. J.; Escobedo, F. A. Macromolecules 2005, 38, 8522-8531.

(40) Škvor, J.; Posel, Z. Macromol. Theory Simul. 2015, 24, 141-151.

(41) Arora, A.; Morse, D. C.; Bates, F. S.; Dorfman, K. D. Soft Matter 2015, 11, 4862-4867.

(42) Fredrickson, G. H.; Binder, K. J. Chem. Phys. 1989, 91, 72657275.

(43) Amundson, K.; Helfand, E. Macromolecules 1993, 26, 13241332.

(44) Lennon, E. M.; Mohler, E. M.; Ceniceros, H. D.; GarciaCervera, C. J.; Fredrickson, G. H. Multiscale Model. Simul. 2008, 6, $1347-1370$

(45) Grzywacz, P.; Qin, J.; Morse, D. C. Phys. Rev. E 2007, 76, 061802.

(46) Koski, J.; Chao, H.; Riggleman, R. A. J. J. Chem. Phys. 2013, 139, 244911.

(47) Glaser, J.; Qin, J.; Medapuram, P.; Müller, M.; Morse, D. C. Soft Matter 2012, 8, 11310-11317.

(48) Gillard, T. M.; Medapuram, P.; Morse, D. C.; Bates, F. S. Macromolecules 2015, 48, 2801-2811. 